\section{Proximal 15q monosomy}

SIR,

We should like to comment upon the letter of Duckett $^{1}$ which criticises our case report of a retarded male with deletion of proximal chromosome 15 and terminal chromosome $10 \mathrm{q}^{2}{ }^{2}$

Firstly, the case is reported as a Short Report, the stipulations for which are that it takes only one page of the journal with no more than three references. It is not meant to be a review of the literature, but to state concisely and simply the salient features of the case. Therefore we did not list all the published papers we referred to which did not show monosomy $10 \mathrm{q}$, but merely cited one reputable book to indicate that we had indeed looked. Also we could not include a lengthy discussion of the relationship of proximal $15 q$ monosomy and the Prader-Willi syndrome (PWS), of which we are well aware, ${ }^{3}$ and as our patient clearly did not have the PWS we referred to "one similar case". We did not profess to review the literature.

Secondly, we submitted our paper to the Editor in February 1981, 6 months before the paper of Duckett and Roberts ${ }^{4}$ appeared and 12 months before the paper of Ledbetter et al. ${ }^{5}$ My previous sentiments remain unchanged, ${ }^{6}$ namely that it is extremely difficult to review the literature 'thoroughly' in a given case. I make the point of referring to cases "known to us" or those "which we have found".

Finally, we do believe that more cases of proximal $15 q-$ are needed to enable an unbiased evaluation of this effect. While an association between PWS and a variety of chromosome 15 abnormalities is apparent, it is not proven that the PWS is 'caused' by proximal $15 \mathrm{q}$ deletion. Hawkey and $\mathrm{Smithies}^{8}$ report four cases of PWS owing to chromosome abnormality of chromosomes other than 15 and Guanti ${ }^{9}$ cites one further case. We found 24 cases of PWS in New South Wales and Victoria with a normal karyotype and two with an abnormal karyotype ${ }^{7}$ (one 47,XXY and one translocation ${ }^{3}$ ). Ledbetter et $a^{5}$ discuss several aspects of this problem and we would like to add our case as an example of proximal 15qproducing abnormalities not of the PWS type, of which one other case with similar abnormalities has also been reported. Duckett and Roberts ${ }^{4}$ themselves state ". . . no consistent phenotypic abnormality has been delineated for partial monosomy 15 making identification of its effects difficult".

Arabella Smith aNd Gesina den DulK
Cytogenetics Unit,
Oliver Latham Laboratory,
Macquarie Hospital,
North Ryde,
NSW 2113,
Australia.

References

1 Duckett D. Proximal 15q monosomy. J Med Genet $1982 ; 19: 393-4$

2 Smith A, Den Dulk G. A severely retarded male with deletion of chromosomes $15(\mathrm{pter} \rightarrow \mathrm{q} 13)$ and $10(\mathrm{q} 26 \rightarrow \mathrm{qter})$. J Med Genet 1982;19:77.

3 Smith A, Noel M. A girl with Prader-Willi syndrome and Robertsonian translocation $45, \mathrm{XX}, \mathrm{t}(14 ; 15) \quad(\mathrm{p} 11 ; \mathrm{q} 11)$ which was present in three normal family members. Hum Genet 1980;55:271-3.

4 Duckett DP, Roberts SH. Adjacent 2 meiotic disjunction. Report of a case resulting from a familial $13 q ; 15 q$ balanced reciprocal translocation and review of the literature. Hum Genet 1982; 58:377-86.

5 Ledbetter DH, Mascarello JT, Riccardi VM, Harper VD, Airhart SD, Strobel RJ. Chromosome 15 abnormalities and the Prader-Willi syndrome: a follow up report of 40 cases. Am J Hum Genet 1982;34:278-85.

- Smith A. Gonadal dysgenesis with 46,X,Xt(qter $\rightarrow$ p221:: p223 $\rightarrow$ qter) karyotype. J Med Genet 1981;18:400.

7 Smith A, Simpson E. Dermatoglyphic analysis of 24 individuals with the Prader-Willi syndrome.J Ment Defic Res 1982;26:91-9.

8 Hawkey CJ, Smithies A. The Prader-Willi syndrome with a 15/15 translocation. Case report and review of the literature. J Med Genet 1976;14:152-63.

9 Guanti G. A new case of re-arrangement of chromosome 15 associated with Prader-Willi syndrome. Clin Genet 1980;17:423-7.

\section{Serum gonadotrophins in Down's syndrome}

SIR,

In the paper entitled 'Serum gonadotrophins in Down's syndrome' (J Med Genet 1982;19:98-9), the authors omitted to mention a study published in $1980^{1}$ in which similar data and conclusions were presented. We feel that our data on 39 patients (both men and women), when added to those reported in the paper under discussion, are mutually supportive and constitute a good point of departure for further studies on this topic. 
Our own study failed to confirm the rise of serum FSH with age. Instead, we found a negative correlation between serum FSH and testicular volume irrespective of age.

\section{J HASEN \\ 25 Lake Street, White Plains, New York 10603, \\ USA.}

\author{
Reference \\ 1 Hasen J, Boyar MR, Shapiro RL. Gonadal function in \\ trisomy 21 . Horm Res 1980;12:345-50.
}

Interstitial duplication/deletion owing to unequal crossing-over in association with pericentric inversion

SIR,

Einfeld and Smith ${ }^{1}$ reported two brothers, aged $4 \frac{1}{2}$ and $2 \frac{1}{2}$, with similar clinical features, namely developmental delay, short stature, and small delicate facies. They were quite different from the other unaffected family members. A pericentric inversion of chromosome 11, inv(11) (p15q14), was found in the younger retarded boy and in the father, whereas the karyotypes of the older retarded boy, the mother, and the two unaffected sibs were normal. Although it was obvious that the clinical features of the two brothers had a similar aetiology, it was not explicable on the grounds of cytogenetic findings. Unknown environmental factors, sex linked mental retardation, or an autosomal recessive condition were considered as possible causes of the abnormal clinical features.

Based on the following personal observation, I would suggest that the clinical features of the two boys may still be explained cytogenetically. Recently, an apparently balanced paracentric inversion of the proximal part of 7q, inv(7) (q11q22), was found in a girl with multiple congenital anomalies and in her phenotypically normal mother. Further examination of high resolution chromosomes revealed an additional tiny dark band on the inverted chromosome 7 of the girl, but not on the inverted chromosome 7 of the mother. It was therefore assumed that an unequal crossing-over had occurred at the base of a meiotic loop of the chromosomes 7 in the mother, which resulted in a tiny interstitial duplication in the girl. A detailed account of this case is published elsewhere. ${ }^{2}$

As suggested in fig 1 , the events of unequal crossing-over occurring in the spermatogenesis of the father, in the two directions shown, would result in identical duplications or deletions either associated

FIG 1 Diagrams illustrating the occurrence and results of unequal crossing-over in the spermatogenesis of the father. (a) Chromosome 11 and its inverted homologue as diagnosed by Einfeld and Smith. The interrupted line denotes the short arm of chromosome 11. (b) Formation of meiotic loops as a consequence of the pericentric inversion. The dotted lines denote the directions of unequal crossing-over. The zigzag lines denote the duplicated or deleted chromosome segment. (c) Various products of unequal crossing-over. Dup $=$ duplication, Del $=$ deletion, $I n v=$ inversion.

with pericentric inversion or not. The older retarded boy could have one of the non-inverted recombinants, and the younger retarded boy one of the inverted recombinants. By performing high resolution chromosome study, the authors may be lucky enough to detect a tiny duplication or deletion in both retarded boys. Nevertheless, the duplicated or deleted chromosome segment might be so tiny that it could not be seen under a microscope.

Unequal crossing-over is a well known event occurring in meiosis. Results of unequal crossingover occurring in the course of evolution are best demonstrated in the anatomy of haemoglobin genes. ${ }^{3}$ For example, the genes for beta, delta, and pseudobeta, which are immediately adjacent to each other within the short arm of chromosome 11, differ only slightly in their base sequences. Haemoglobinopathies such as haemoglobin Lepore with gene 